\title{
Analyzing Dorian Twitter data to understand how hurricane risk communication changes as threats unfold
}

\author{
Qian Ma and Barbara Millet \\ University of Miami
}

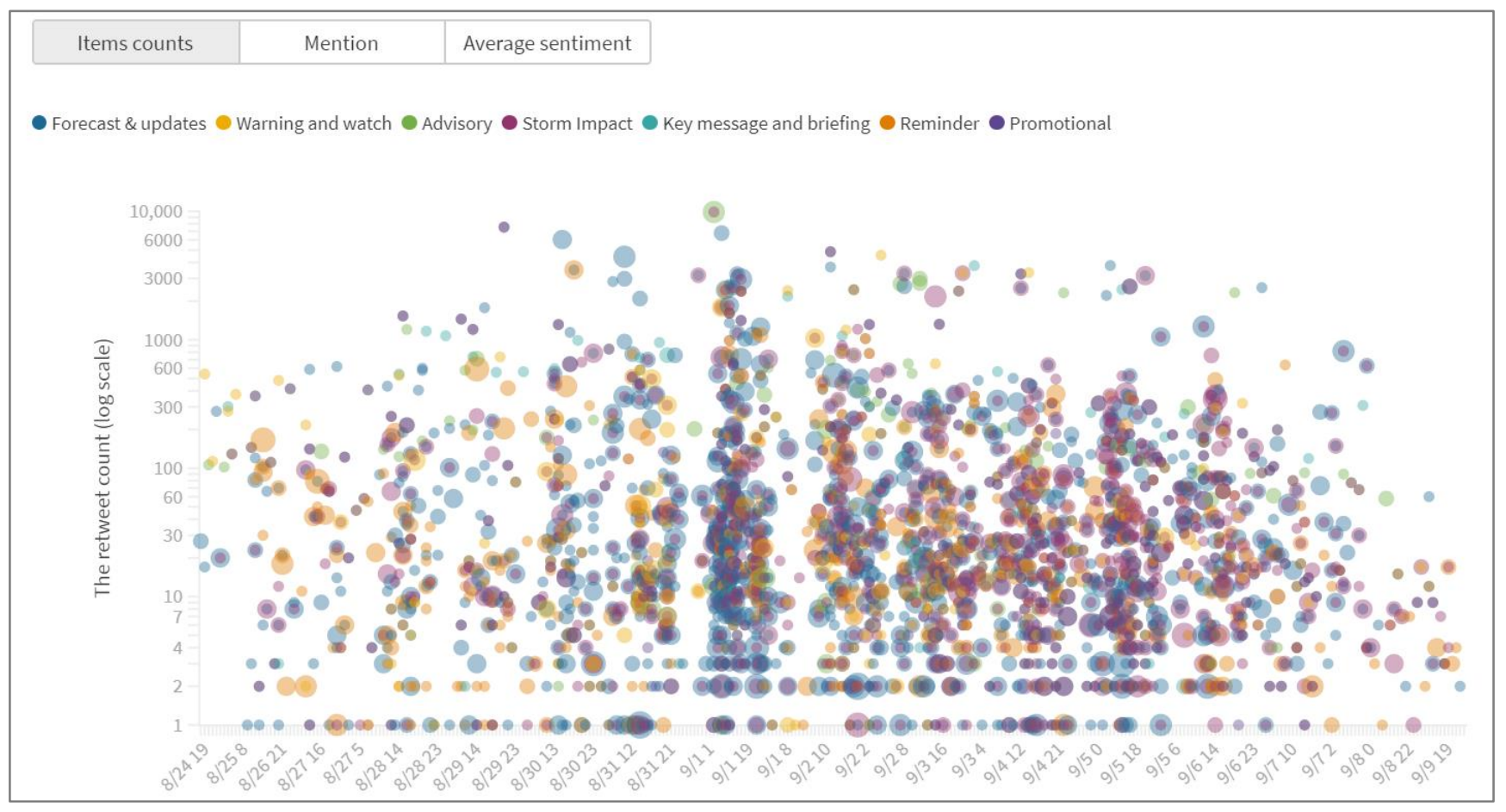

\section{ABOUT THE VISUAL CASE STUDY}

Communicating effective hurricane risk messages on social media presents challenges. Previous research efforts have used Twitter data to examine hurricane risk communication patterns across different hurricane phases (before, during, and after) and communicator engagement (how authorities distribute information and the public's reactions). While these studies provide insight into hurricane risk communication on social media, how messages are disseminated through hurricanerelated social networks on Twitter is unknown. Hence, this research explores diffusion of forecast messages on Twitter to understand how hurricane risk information is created, disseminated, and discussed in many-to-many communication modes.

We collected both text- and image-based hurricane risk messages from tweets posted for Hurricane Dorian, the first major hurricane during the 2019 Atlantic hurricane season. Using Twitter REST (Representational State Transfer) and stream API (application program interface) via the R-Twitter package, we collected 21,775 tweets from 555 accounts posted between August 20 and September 10, 2019. The accounts included 283 organizational and 272 professional accounts, and the 21,775 posts included 10,141 original tweets $(3,569$ from organizations), 6,268 replies (2,721 from organizations), and

*(qxm70,bmillet)@miami.edu.
5,366 retweets (1,558 from organizations). Based on the streams, we were able to study the diffusion of risk messaging and post sentiment. We conducted descriptive statistical analysis of manually coded tweets of professional hurricane risk messages, network analysis of diffusion patterns among the messages and their creators, and sentiment analysis of original messages and user replies.

In this visual case study, we present 9 visualizations exploring message diffusion and sentiment of Hurricane Dorian Twitter Data. The interactive demo of the visualizations is available at: https://public.flourish.studio/story/484742/. Results show that professional individual accounts are more likely to post original content than share, with no observable temporal regulation, in variable formats, and with mixed sentiment. In contrast, most of the organizational accounts (including forecasters, emergency management, and news outlets) post in regular intervals with a fixed message format and a neutral opinion. Additionally, engagement across forecaster accounts is lower than engagement across mediator accounts (i.e. news outlets and professional individual account holders). The study also revealed that within the hurricane risk context, those willing to share information rely more on post timing and expertise of post creators than on message type and sentiment. This research suggests that increasing engagement across the primary accounts may improve the effectiveness of hurricane risk communication across the hurricane warning system. 